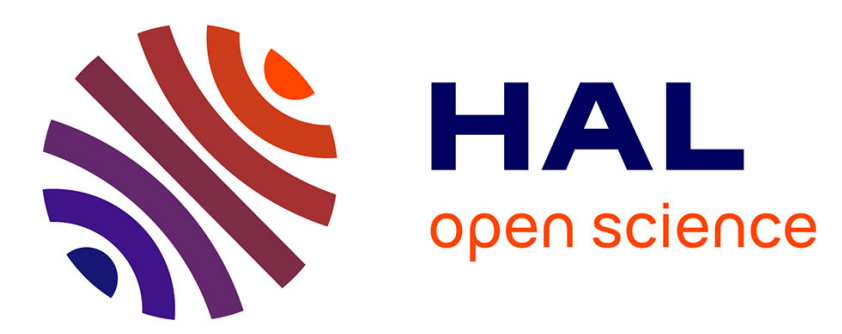

\title{
VT Scan: Towards an Efficient Pipeline from Computed Tomography Images to Ventricular Tachycardia Ablation
}

Nicolas Cedilnik, Josselin Duchateau, Rémi Dubois, Pierre Jaïs, Hubert Cochet, Maxime Sermesant

\section{- To cite this version:}

Nicolas Cedilnik, Josselin Duchateau, Rémi Dubois, Pierre Jaïs, Hubert Cochet, et al.. VT Scan: Towards an Efficient Pipeline from Computed Tomography Images to Ventricular Tachycardia Ablation. Functional Imaging and Modelling of the Heart, Jun 2017, Toronto, Canada. pp.271-279, 10.1007/978-3-319-59448-4_26. hal-01498672v2

\section{HAL Id: hal-01498672 \\ https://hal.inria.fr/hal-01498672v2}

Submitted on 3 Apr 2017

HAL is a multi-disciplinary open access archive for the deposit and dissemination of scientific research documents, whether they are published or not. The documents may come from teaching and research institutions in France or abroad, or from public or private research centers.
L'archive ouverte pluridisciplinaire HAL, est destinée au dépôt et à la diffusion de documents scientifiques de niveau recherche, publiés ou non, émanant des établissements d'enseignement et de recherche français ou étrangers, des laboratoires publics ou privés. 


\title{
VT Scan: Towards an Efficient Pipeline from Computed Tomography Images to Ventricular Tachycardia Ablation
}

\author{
N. Cedilnik ${ }^{1}$, J. Duchateau ${ }^{2}$, R. Dubois ${ }^{2}$, P. Jaïs ${ }^{2}$, H. Cochet $^{2}$, and \\ M. Sermesant ${ }^{1}$ \\ 1 Université Côte d'Azur, Inria, France \\ ${ }^{2}$ Liryc Institute, Bordeaux, France
}

\begin{abstract}
Non-invasive prediction of optimal targets for efficient radiofrequency ablation is a major challenge in the treatment of ventricular tachycardia. Most of the related modelling work relies on magnetic resonance imaging of the heart for patient-specific personalized electrophysiology simulations.

In this study, we used high-resolution computed tomography images to personalize an Eikonal model of cardiac electrophysiology in seven patients, addressed to us for catheter ablation in the context of postinfarction arrhythmia. We took advantage of the detailed geometry offered by such images, which are also more easily available in clinical practice, to estimate a conduction speed parameter based on myocardial wall thickness. We used this model to simulate a propagation directly on voxel data, in similar conditions to the ones invasively observed during the ablation procedure.

We then compared the results of our simulations to dense activation maps that recorded ventricular tachycardias during the procedures. We showed as a proof of concept that realistic re-entrant pathways responsible for ventricular tachycardia can be reproduced using our framework, directly from imaging data.
\end{abstract}

Keywords: Electrophysiological modelling - Heart imaging · Ventricular tachycardia $\cdot$ Catheter ablation

\section{Introduction}

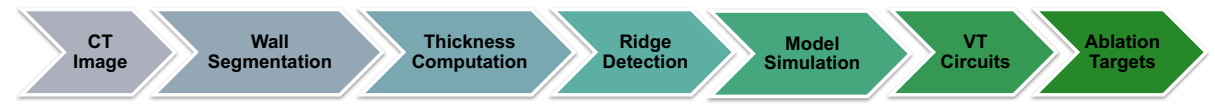

Fig. 1. Overall pipeline of the approach. Ridge and circuit detection are not presented in this article. 
Sudden cardiac death (SCD) due to ventricular arrhythmia is responsible for hundreds of thousands of deaths each year [1]. A high proportion of these arrhythmias are related to ischemic cardiomyopathy, which promotes both ventricular fibrillation and ventricular tachycardia (VT). The cornerstone of SCD prevention in an individual at risk is the use of an implantable cardioverter defibrillator (ICD). ICDs reduce mortality, but recurrent VT in recipients are a source of important morbidity. VT causes syncope, heart failure, painful shocks and repetitive ICD interventions reducing device lifespan. In a small number of cases called arrhythmic storms, the arrhythmia burden is such that the ICD can be insufficient to avoid arrhythmic death.

In this context, radio-frequency ablation aiming at eliminating re-entry circuits responsible for VT has emerged as an interesting option. VT ablation has already demonstrated its benefits [2] but still lacks clinical consensus on optimal ablation strategy [3]. Two classical strategies have been developped by electrophysiologists. The first strategy focuses on mapping the arrhythmia circuit by inducing VT before ablating the critical isthmus. The second strategy focuses on the substrate, eliminating all abnormal potentials that may contain such isthmuses.

Both strategies are very time-consuming, with long mapping and ablation phases respectively, and procedures are therefore often incomplete in patients with poor general condition. Many authors have thus shown an interest in developing methods coupling non-invasive exploration and modelling to predict both VT risk and optimal ablation targets [4-8]. Most of the published work in this area relies on cardiac magnetic resonance imaging (CMR), at the moment considered as the gold standard to assess myocardial scar, in particular using late gadolinium enhancement sequences. However, despite being the reference method to detect fibrosis infiltration in healthy tissue, CMR methods clinically available still lack spatial resolution under $2 \mathrm{~mm}$ to accurately assess scar heterogeneity in chronic healed myocardial infarction, because the latter is associated with severe wall thinning (down to $1 \mathrm{~mm}$ ). Moreover, most patients recruited for VT ablation cannot undergo CMR due to an already implanted ICD.

In contrast, recent advances in cardiac computed tomography (CT) technology now enable the assessment of cardiac anatomy with extremely high spatial resolution. We hypothesized that such resolution could be of value in assessing the heterogeneity of myocardial thickness in chronic healed infarcts. Based on our clinical practice, we indeed believe that detecting VT isthmuses relies on detecting thin residual layers of muscle cells-rich tissue inside infarct scars that CMR fails to identify due to partial volume effect. CT image acquisistion is less operator-dependent than CMR, making it a first class imaging modality for automated and reproductible processing pipelines. CT presents fewer contraindication than CMR (it is notably feasible in patients with ICD), it costs less, and its availability is superior.

The aim of this study was to assess the relationship between wall thickness heterogeneity, as assessed by CT, and ventricular tachycardia mechanisms in chronic myocardial infarction, using a computational approach (see the overall 
pipeline in Fig. 1). This manuscript presents the first steps of this pipeline, in order to evaluate the relationship between simulations based on wall thickness and electro-anatomical mapping data.

\section{Image Acquisition and Processing}

\subsection{Population}

The data we used come from 7 patients (age $58 \pm 7$ years, 1 woman) referred for catheter ablation therapy in the context of post-infarction ventricular tachycardia. The protocol of this study was approved by the local research ethics committee.

\subsection{Acquisition}

All the patients underwent contrast-enhanced ECG-gated cardiac multi-detector CT (MDCT) using a 64-slice clinical scanner (SOMATOM Definition, Siemens Medical Systems, Forchheim, Germany) between 1 to 3 days prior to the electrophysiological study. This imaging study was performed as part of standard care as there is an indication to undergo cardiac MDCT before electrophysiological procedures to rule out intra cardiac thrombi. Coronary angiographic images were acquired during the injection of a $120 \mathrm{ml}$ bolus of iomeprol $400 \mathrm{mg} / \mathrm{ml}$ (Bracco, Milan, Italy) at a rate of $4 \mathrm{ml} / \mathrm{s}$. Radiation exposure was typically between 2 and $4 \mathrm{mSv}$. Images were acquired in supine position, with tube current modulation set to end-diastole. The resulting voxels have a dimension of $0.4 \times 0.4 \times 1 \mathrm{~mm}^{3}$.

\subsection{Segmentation}
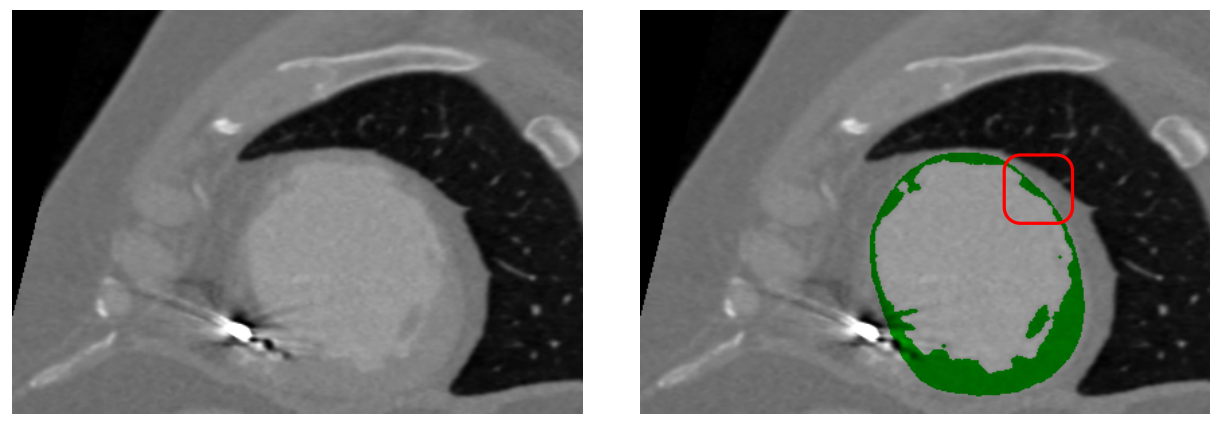

Fig. 2. (Left) Original CT-scan image. (Right) Wall mask (green). Note the thickness heterogeneity (red box).

The left ventricular endocardium was automatically segmented using a regiongrowing algorithm, the thresholds to discriminate between the blood pool and 
the wall being optimized from a prior analysis of blood and wall densities. The epicardium was segmented using a semi-automated tool based on the interpolation of manually-drawn polygons.

All analyses were performed using the MUSIC software ${ }^{3}$ (IHU Liryc Bordeaux, Inria Sophia Antipolis, France). An example of the result of such segmentation can be seen in Fig. 2 .

\subsection{Wall Thickness Computation}
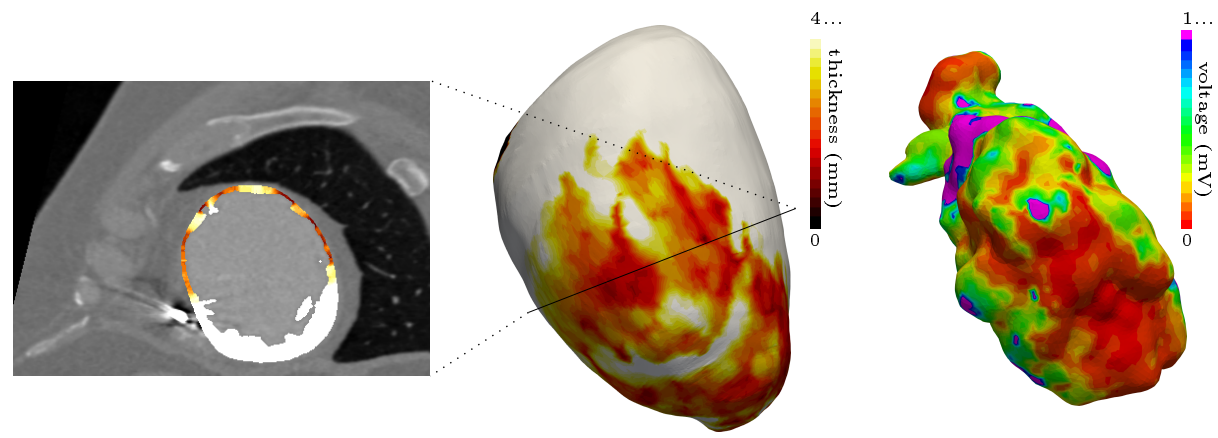

Fig. 3. (Left-Middle) Wall thickness as defined in section 2.4 (Right) Voltage map recorded during sinus rhythm.

In CT-scan images, only the healthy part of the myocardium is visible, hence wall thinning is a good marker of scar localization and abnormal electrophysiological parameters $[9]$. To accurately compute wall thickness, and to overcome difficulties related to its definition on tri-dimensional images, we chose the Yezzi et al. method [10]. It is based on solving the Laplace equation with Dirichlet boundary conditions to determine the trajectories along which the thickness will be computed. One advantage of such thickness definition is that it is then defined for every voxel, which is later useful in our model (see section 3.2). An example of the results can be observed in Fig. 3 (left and middle), along with a comparison with the much lower level of detail in scar morphology assessment that is obtained with a voltage map from a sinus rhythm recording.

\section{Cardiac Electrophysiology Modelling}

\subsection{Eikonal Model}

We chose the Eikonal model for simulating the wave front propagation because of the following reasons:

\footnotetext{
3 https://team.inria.fr/asclepios/software/music/
} 
- It requires very few parameters compared with biophysical models, making it more suitable for patient-specific personalization in a clinical setting.

- Its output is an activation map directly comparable with the clinical data.

- It allows for very fast solving thanks to the fast marching algorithm.

We used the standard Eikonal formulation in this study:

$$
v(X)|| \nabla T(X)||=1
$$

The sole parameter required besides the myocardial geometry is the wave front propagation speed $v(X)$ (see section 3.2). More sophisticated biophysical models allow for more precise results; however, this precision relies on an accurate estimate of the parameters and the necessary data to such ends are not available in clinical practice.

This original approach enabled us to parametrize directly the model on the basis of the thickness computed from the images. Additionally, we generated a unidirectional onset by creating an artificial conduction block. This block was needed to represent the refractoriness of the previously activated tissue that the Eikonal model does not include. An illustration of this phenomenon can be observed in the green box in Fig. 4 .

\subsection{Wave Front Propagation Speed Estimation}
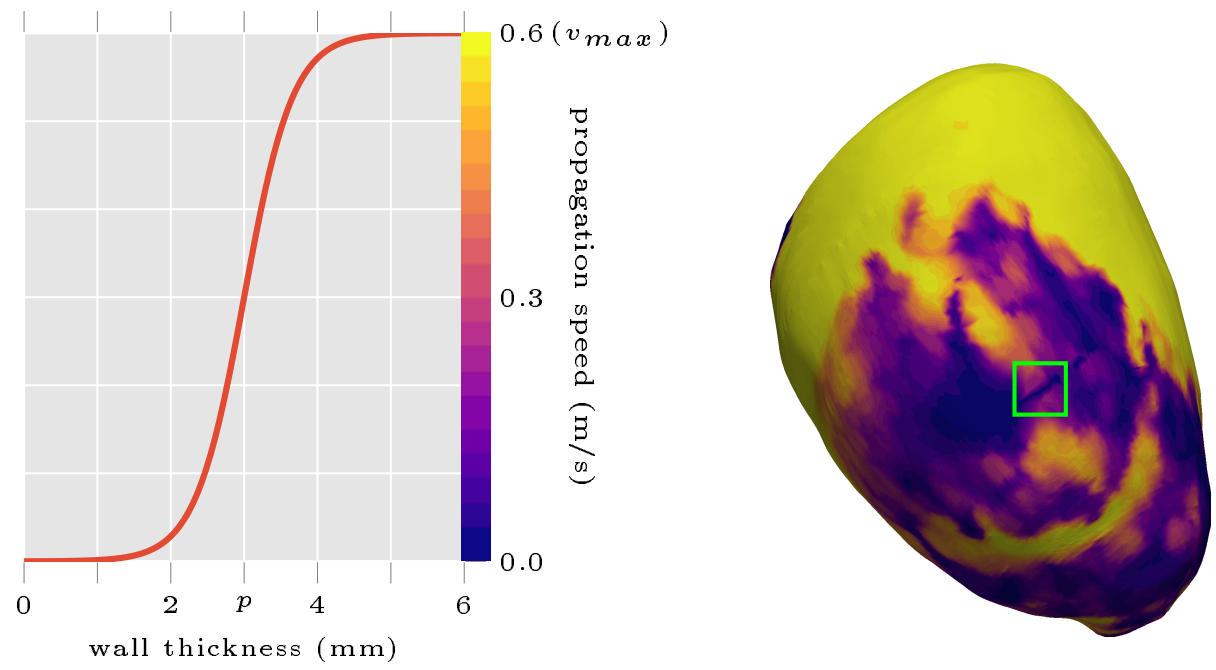

Fig. 4. (Left) Transfer function used to estimate wave front propagation speed from wall thickness. (Right) Example resulting speed map. Note the artificial refractory block (dark straight line in green box). 
As there is a link between myocardial wall thickness and its viability, it was possible to parameterize our model from the previous wall thickness computation (see section 2.4). There are basically three different cases:

1. Healthy myocardium where the wave front propagation speed is normal.

2. Dense fibrotic scar where the wave front propagation is extremely slow.

3. Gray zone area where it is somewhere in between.

Instead of arbitrarily choosing a specific speed for the "gray zone", we exploited the resolution offered by our images to come up with a smooth, continuous estimate of the speed between healthy and scar myocardium. This resulted in the following logistic transfer function:

$$
v(X)=\frac{v_{\max }}{1+e^{r(p-t(X))}}
$$

where $v_{\max }$ is the maximum wave front propagation speed, $t(X)$ the thickness at voxel $X, p$ the inflection point of the sigmoidal function, i.e., the thickness at which we reach $\frac{1}{2} v_{\max }$ and $r$ a dimensionless parameter defining the steepness of the transfer function.

More specifically, we chose the following parameters:

- $v_{\max }=0.6 \mathrm{~m} / \mathrm{s}$, as it is the conduction speed of healthy myocardium,

- $p=3 \mathrm{~mm}$, as it was considered the gray zone "center",

$-r=2$, in order to obtain virtually null speed in areas where thickness is below $2 \mathrm{~mm}$.

The resulting transfer function can be visualized in Fig. 4

The fiber orientations were not included in our simulations. They might be in our final pipeline, but it is worth noting that we obtained satisfying results without this parameter.

\section{Electrophysiological Data}

In order to evaluate the simulation results, we compared them to electro-anatomical mapping data. As part of the clinical management of their arrhythmia, all the patients underwent an electrophysiological procedure using a 3-dimensional electroanatomical mapping system (Rhythmia, Boston scientific, USA) and a basket catheter (Orion, Boston scientific, USA) dedicated to high-density mapping. During the procedure, ventricular tachycardia was induced using a dedicated programmed stimulation protocol, and the arrhythmia could be mapped at extremely high density (about 10000 points per map). Patients were then treated by catheter ablation targeting the critical isthmus of the recorded tachycardia, as well as potential other targets identified either by pace mapping or sinus rhythm substrate mapping. These maps were manually registered to the CT images. 

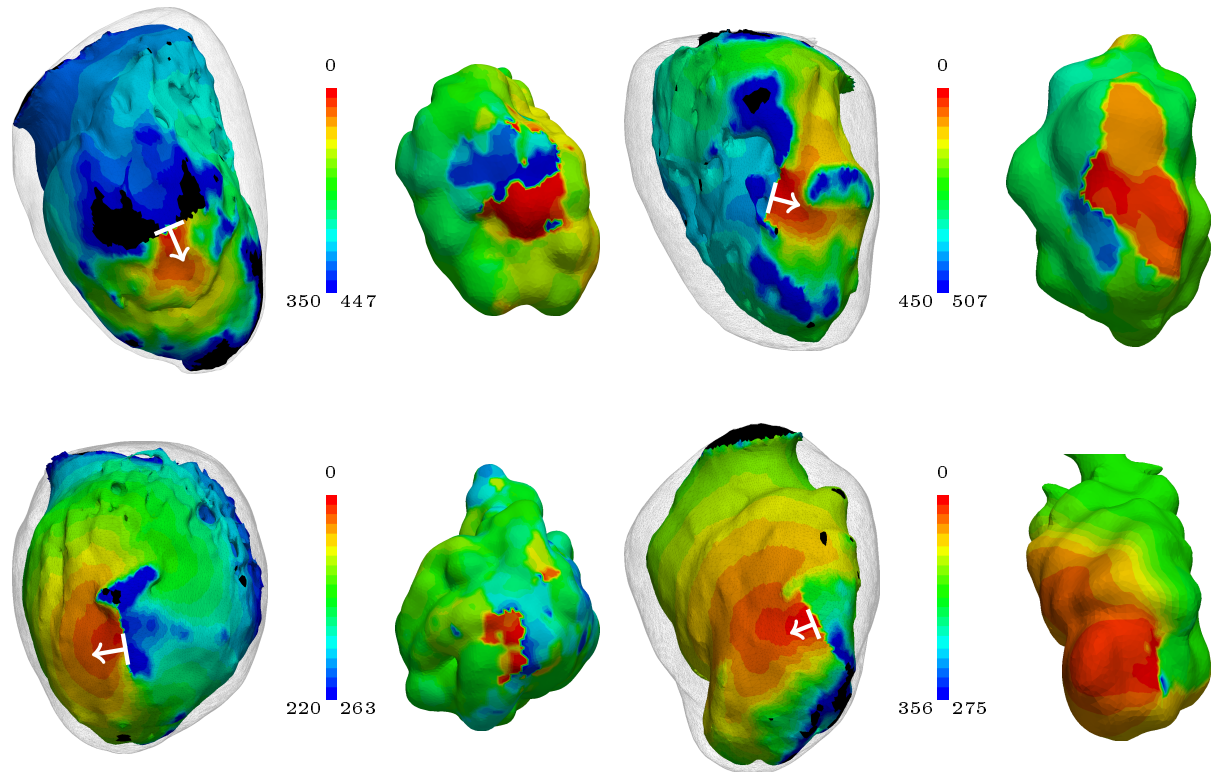

Fig. 5. Comparison of predicted (left) activation maps (in ms) to "ground truth" data (right) obtained from VT recording during RF catheter ablation in 4 different patients. White arrows: starting point and direction of simulated electrical impulse. Both recorded and simulated VT represent one cycle only. Animations of these activation maps are available online ${ }^{5}$

\section{$5 \quad$ Results}

Examples results of the simulations and their comparisons to mapping data are presented in Fig. 5 In each case, a qualitatively similar re-entry circuit is predicted.

Similar visual results were reached for all the VTs, without any further tuning of the model. However, to reach such results we needed to pick the stimulations points and directions very carefully.

\section{Implementation}

All the activation maps acquired during ventricular tachycardia (10 maps of 10 different circuits acquired in 7 patients) were exported to Matlab software (Mathworks, USA).

The simulation was computed directly on the voxel data as the resolution of our images represents highly detailed anatomy, and to avoid arbitrary choices inherent to mesh construction. We then mapped the results to a mesh but for visualization purposes only.

\footnotetext{
5 https://team.inria.fr/asclepios/vt-scan-fimh-2017/
} 
We used a custom Python packag 6 for the thickness computation and the SimpleITK fast marching implementation to solve the Eikonal equation.

The artificial conduction block was created by setting the wave front propagation speed to zero in a parametrically defined disk of $15 \mathrm{~mm}$ radius, $2 \mathrm{~mm}$ behind the stimulation points, orthogonal to the desired stimulation direction.

From the results of the semi-automatic CT image segmentation, the complete simulation pipeline, with an informal benchmark realized on a i7-5500U CPU at $2.40 \mathrm{GHz}$ (using only 1 core), looks as follows:

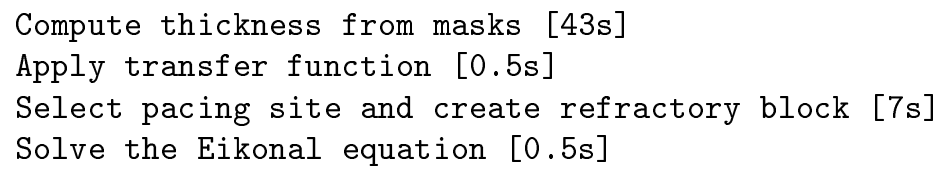

\section{Discussion}

This article presents a framework that may be suitable for VT RF ablation targets and prediction of VT risk in daily clinical practice. The results presented here are preliminary but promising, due to the robustness of the image processing, the fast simulation of the model, and the high-resolution of CT scan images. This resolution was crucial in the characterization of the VT isthmuses presented in this study.

The main limitations of the results presented here are the limited sample size and the lack of quantitative evaluation of the simulation results. It is however worth noting that we were able to obtain such results without advanced calibration of the model's parameters. For the latter, we plan to map the electrophysiological data onto the image-based meshes for more quantitative comparison. However it remains challenging due to the shape differences. Heterogeneity in $\mathrm{CT}$ images also induce a bias in thickness computation that may require patientspecific personnalization of the transfer function parameters.

We believe that in order to fully automatize the pipeline, we need not only to determine a ridge detection strategy, but also to overcome some of the simplifications induced by the Eikonal modelling choice and enhance the circuit characterization.

\section{References}

1. D. Mozaffarian, E. J. Benjamin, A. S. Go, D. K. Arnett, M. J. Blaha, M. Cushman, S. R. Das, S. de Ferranti, J.-p. Desprs, H. J. Fullerton, et al. "Executive summary: Heart Disease and Stroke Statistics-2016 update: A report from the American Heart Association." In: Circulation 133.4 (2016), p. 447.

$6 \longdiv { \text { https://pypi.python .org/pypi/pyezzi } }$ 
2. H. Ghanbari, K. Baser, M. Yokokawa, W. Stevenson, P. Della Bella, P. Vergara, T. Deneke, K.-H. Kuck, H. Kottkamp, S. Fei, et al. "Noninducibility in Postinfarction Ventricular Tachycardia as an End Point for Ventricular Tachycardia Ablation and Its Effects on Outcomes". In: Circulation: Arrhythmia and Electrophysiology 7.4 (2014), pp. 677-683.

3. E. M. Aliot, W. G. Stevenson, J. M. Almendral-Garrote, F. Bogun, C. H. Calkins, E. Delacretaz, P. Della Bella, G. Hindricks, P. Jas, M. E. Josephson, et al. "EHRA/HRS expert consensus on catheter ablation of ventricular arrhythmias". In: Europace 11.6 (2009), pp. 771-817.

4. H. Ashikaga, H. Arevalo, F. Vadakkumpadan, R. C. Blake, J. D. Bayer, S. Nazarian, M. M. Zviman, H. Tandri, R. D. Berger, H. Calkins, et al. "Feasibility of image-based simulation to estimate ablation target in human ventricular arrhythmia". In: Heart Rhythm 10.8 (2013), pp. 1109-1116.

5. H. Arevalo, G. Plank, P. Helm, H. Halperin, and N. Trayanova. "Tachycardia in post-infarction hearts: insights from 3D image-based ventricular models". In: PloS one 8.7 (2013), e68872.

6. R. Cabrera-Lozoya, J. Margeta, L. Le Folgoc, Y. Komatsu, B. Berte, J. Relan, H. Cochet, M. Haissaguerre, P. Jais, N. Ayache, et al. "Confidence-based training for clinical data uncertainty in image-based prediction of cardiac ablation targets". In: International MICCAI Workshop on Medical Computer Vision. Springer. 2014, pp. 148-159.

7. Z. Chen, R. Cabrera-Lozoya, J. Relan, M. Sohal, A. Shetty, R. Karim, H. Delingette, J. Gill, K. Rhode, N. Ayache, et al. "Biophysical Modeling Predicts Ventricular Tachycardia Inducibility and Circuit Morphology: A Combined Clinical Validation and Computer Modeling Approach". In: Journal of cardiovascular electrophysiology 27.7 (2016), pp. 851-860.

8. L. Wang, O. A. Gharbia, B. M. Horek, and J. L. Sapp. "Noninvasive epicardial and endocardial electrocardiographic imaging of scar-related ventricular tachycardia". In: Journal of Electrocardiology 49.6 (2016), pp. 887-893.

9. Y. Komatsu, H. Cochet, A. Jadidi, F. Sacher, A. Shah, N. Derval, D. Scherr, P. Pascale, L. Roten, A. Denis, et al. "Regional myocardial wall thinning at multi-detector computed tomography correlates to arrhythmogenic substrate in post-infarction ventricular tachycardia: assessment of structural and electrical substrate". In: Circulation: Arrhythmia and Electrophysiology (2013), CIRCEP-113.

10. A. J. Yezzi and J. L. Prince. "An Eulerian PDE approach for computing tissue thickness". In: IEEE transactions on medical imaging 22.10 (2003), pp. $1332-1339$. 